\title{
Dynamic Transfer Reference Point Oriented MOEA/D Involving Local Objective-Space Knowledge
}

\author{
Yingbo Xie, Shengxiang Yang, Senior Member, IEEE, Ding Wang, Senior Member, IEEE, \\ Junfei Qiao, Senior Member, IEEE, and Baocai Yin, Member, IEEE
}

\begin{abstract}
The decomposition-based multi-objective evolutionary algorithm (MOEA/D) has attained excellent performance in solving optimization problems involving multiple conflicting objectives. However, the Pareto optimal front (POF) of many multi-objective optimization problems (MOPs) has irregular properties, which weakens the performance of MOEA/D. To address this issue, we devise a dynamic transfer reference point oriented MOEA/D with local objective-space knowledge (DTRMOEA/D). The design principle is based on three original and rigorous mechanisms. First, the individuals are projected onto a line segment (two-objective case) or a three-dimensional plane (three-objective case) after being normalized in the objective space. The line segment or the plane is divided into three different regions: the central region, the middle region, and the edge region. Second, a dynamic transfer criterion of reference point is developed based on population density relationships in different regions. Third, a strategy of population diversity enhancement guided by local objective-space knowledge is adopted to improve the diversity of the population. Finally, the experimental results conducted on sixteen benchmark MOPs and eight modified MOPs with irregular POF shapes verify that the proposed DTRMOEA/D has attained a strong competitiveness compared with other representative algorithms.
\end{abstract}

Index Terms-Multi-objective optimization, decomposition, dynamic transfer reference point, local objective space.

\section{INTRODUCTION}

$\mathbf{I}$ $\mathrm{N}$ scientific research and engineering practice, the problem consisting of multiple conflicting objective functions that need to take the minimum or maximum simultaneously is often called multi-objective optimization problem [1]-[8]. The population-based multi-objective evolutionary algorithms aim at obtaining a range of trade-off solutions among multiple conflicting objectives [9]-[11]. The nature of multi-objective evolutionary algorithms (MOEAs) enable them to achieve excellent performance when dealing with multi-objective optimization problems. Common examples of real-world multiobjective optimization problems include multi-objective op-

This work was supported in part by the Beijing Natural Science Foundation under Grant JQ19013, in part by the National Natural Science Foundation of China under Grant 61773373, Grant 61890930-5, Grant 61533002, and Grant 62021003, and in part by the National Key Research and Development Project under Grant 2018YFC1900800-5. (Corresponding author: Junfei Qiao)

Yingbo Xie, Ding Wang, Junfei Qiao, and Baocai Yin are with the Faculty of Information Technology, Beijing University of Technology, Beijing 100124, China (email: xieyingbo@emails.bjut.edu.cn; dingwang@bjut.edu.cn; junfeiq@bjut.edu.cn; ybc@bjut.edu.cn).

Shengxiang Yang is with the Centre for Computational Intelligence, School of Computer Science and Informatics, De Montfort University, The Gateway, Leicester LE1 9BH, U.K. (email: syang@dmu.ac.uk). timal control of urban wastewater treatment processes [12], [13], teaching manipulator [14], barrier coverage with wireless sensors [15], image feature extraction in the presence of noise [16], the design of a trauma system [17], and risk-based optimal operation of a hybrid energy system [18].

For the sake of more precise description, it is imperative to give a unified definition of the multi-objective optimization problem (MOP) studied in this paper. A minimized MOP can be formulated as follows:

$$
\begin{array}{ll}
\operatorname{minimize} & \mathbf{f}(\mathbf{x})=\left(f_{1}(\mathbf{x}), f_{2}(\mathbf{x}), \cdots, f_{M}(\mathbf{x})\right)^{\top} \\
\text { subject to } & \mathbf{x} \in \Omega
\end{array}
$$

where $\mathbf{x}=\left(x_{1}, x_{2}, \cdots, x_{D}\right) \in \Omega$ is a decision vector consisting of $D$ decision variables, and $\Omega \subseteq \mathbb{R}^{D}$ is the decision space. $\mathrm{f}: \Omega \longrightarrow \Gamma \subseteq \mathbb{R}^{M}$ defines $M$ objective functions, and $\Gamma$ is the objective space. Assuming that $\mathbf{x}^{a}$ and $\mathbf{x}^{b}$ are two different solutions, $\mathbf{x}^{a}$ dominant $\mathbf{x}^{b}$ needs to meet the following two conditions:

$$
\left\{\begin{array}{l}
f_{k}\left(\mathbf{x}^{a}\right) \leq f_{k}\left(\mathbf{x}^{b}\right), \forall k \in\{1,2, \cdots, M\} \\
f_{j}\left(\mathbf{x}^{a}\right)<f_{j}\left(\mathbf{x}^{b}\right), \exists j \in\{1,2, \cdots, M\} .
\end{array}\right.
$$

A solution $\mathbf{x}^{c} \in \Omega$ is considered to be Pareto optimal if and only if no other solution $\mathbf{x}^{d} \in \Omega$ can dominate $\mathbf{x}^{c}$. The set of all Pareto optimal solutions is defined as the Pareto optimal set (POS). The dominance relation in the decision space is consistent with the dominance relation in the objective space, so the solution set which is composed of the objective function values corresponding to the POS is called Pareto optimal front (POF).

Due to the excellent performance in solving MOPs, MOEAs with different characteristics have sprung up in a variety of theoretical research and engineering applications. According to different evolutionary criteria, most MOEAs can be classified into three representative categories [19].

The first category covers the MOEAs employing the Pareto dominance-based criterion to select the candidate solutions and meet the evolving needs of the population [20], [21]. The nondominated sorting genetic algorithm II (NSGA-II) was proposed to improve population synthesis performance while reducing the computational complexity [22]. An improved strength Pareto evolutionary algorithm (SPEA2) was designed to select suitable non-dominant individuals for mating selection [23]. An effective two-archive strategy to solve the problem of convergence and diversity imbalance was given 
in [24]. An external elite archive strategy based on convergence information was integrated into the MOEA framework to accelerate the convergence process [25]. Furthermore, an efficient non-dominated sort approach was studied in [26]. It is believed that the non-dominated sorting strategy will still be a hot topic of MOEAs.

The second category is known as the indicator-based MOEAs, where performance indicators are used to guide the optimization process. A binary performance indicator for the selection process was defined in [27]. An enhanced inverted generational distance indicator was adopted as a measure of contribution to promote the convergence speed of individuals [28]. A simple hypervolume indicator was also applied to update the exact contribution of different individuals [29]. The Divergence theorem-based R2 indicator was derived for better hypervolume approximation [30]. Moreover, a new R2 indicator was selected to assist the MOEA to solve MOPs [31]. An indicator-based MOEA with diversity enhancement strategy for large-scale multiobjective optimization was described in [32]. It is worth investigating to incorporate the evolutionary state information of the population or the preference information of decision-makers into the indicator-based MOEAs.

The third category refers to the decomposition-based MOEAs [33]. MOEA/D was developed to transform an MOP into a set of scalar optimization subproblems and to find the solution of each subproblem by using the information of its neighboring subproblems [34]. The distribution of weight vectors has a great correlation with the distribution of individuals, so people have been holding great enthusiasm for the research of improving the distribution of weight vectors [35]. A novel weight vector initialization and distribution strategy was embedded into the original MOEA/D framework to solve MOPs with complex POF shapes [36]. An adaptive weight vector guided MOEA was presented in [37] to obtain a set of uniformly distributed optimal individuals. The preference information was embedded into the MOEA/D framework to improve its performance [38]. A systematic approach was presented to generate weight vectors for multi-objective optimization [39]. In addition, a generalized adaptive adjustment strategy of weight vector was given in [40]. Furthermore, two sets of weights were used to balance convergence and diversity during the evolutionary process [41]. A two-stage strategy was adopted in which the ideal point was set as the initial reference point, and whether to change the reference point was judged when the evolution generations reach a certain set value [42]. The above two improvements have promoted the performance of MOEA/D to some extent. However, the number of subproblems assigned to each reference point or the number of evolutionary generations in the first evolutionary stage was artificially set and was not related to the evolutionary state of the population.

To effectively address these shortcomings, a dynamic transfer reference point oriented MOEA/D with local objectivespace knowledge, DTR-MOEA/D for short, is proposed in this paper. We highlight the main novelty and contributions of our proposed DTR-MOEA/D compared with recently proposed representative algorithms as follows.

1) The individuals in the population generated after each iteration are normalized in the objective space. The normalized individuals are projected onto a line segment or a three-dimensional plane. More specifically, in the case of two objectives, the normalized individuals are projected onto a line segment. We take the maximum of two objectives as the two endpoints of the line segment and then the line segment is divided into six segments equidistantly. The two adjacent segments located in the center are marked as the central region, the two segments at the two ends are marked as the edge region, and the remaining two segments are recorded as the middle region. The number of individuals in the population projected onto each region is calculated respectively. In the case of three objectives, the normalized individuals are projected onto a three-dimensional plane. It is worth noting that for the convenience of calculation, the areas of different regions are not equal, which is different from the situation of the bi-objective case. The number of individuals in the population projected onto each region is calculated respectively.

2) A reasonable reference point dynamic transfer criterion is formulated. According to the number of individuals projected onto different regions, the density relationship of individuals in adjacent regions is judged, and the position of the reference point in the next iteration is dynamically adjusted according to the density relationship.

3) A diversity enhancement mechanism guided by individual density knowledge in local objective-space is designed. The minimum value of the angle between each individual and the remaining individuals in global objective-space is obtained, and the average value of the $\mathrm{N}$ minimum values $(\mathrm{N}$ represents the number of individuals in the population) is calculated and recorded as the global angle average value. We calculate the minimum angle value between each individual and the rest individuals in the local objective-space, and calculate the average value of the angles, which is recorded as the local angle average value. The appropriate mating individuals are selected by judging the relationship between the global angle average and the local angle average.

The rest of this paper is outlined as follows. Section II introduces the fundamental concepts and discusses the existing works. The details of the proposed DTR-MOEA/D are presented in Section III. Section IV studies the parameter setting, presents the experimental results on benchmark MOPs and modified MOPs with irregular Pareto front shapes, and compares the proposed DTR-MOEA/D with other representative MOEAs. Finally, the conclusions are drawn in Section V, and in addition, future works are illustrated in this section.

\section{Preliminaries And Related Works}

\section{A. Penalty-based Boundary Intersection Approach}

MOEA/D transforms an MOP into a set of scalar optimization subproblems by using a basic decomposition strategy and then optimizes them simultaneously. In MOEA/D, each subproblem is optimized mainly by its neighborhood information. Among the various MOEA/D variants, commonly used 


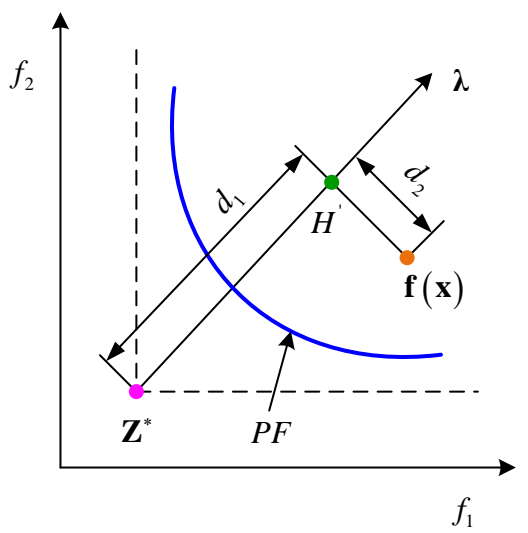

Fig. 1. Illustration of PBI approach.

decomposition methods include the weighted sum approach, Tchebycheff approach, and penalty-based boundary intersection approach.

The penalty-based boundary intersection approach is used in the proposed DTR-MOEA/D, and a scalar optimization subproblem can be defined mathematically as follows:

$$
\begin{aligned}
& \min g^{\mathrm{pbi}}\left(\mathbf{x} \mid \boldsymbol{\lambda}, \boldsymbol{z}^{*}\right)=d_{1}+\theta d_{2} \\
& \text { subject to } \quad \mathbf{x} \in \Omega
\end{aligned}
$$

where

$$
\begin{gathered}
d_{1}=\frac{\left\|\left(\mathbf{f}(\mathbf{x})-\boldsymbol{z}^{*}\right)^{\top} \boldsymbol{\lambda}\right\|}{\|\boldsymbol{\lambda}\|} \\
d_{2}=\left\|\mathbf{f}(\mathbf{x})-\left(\boldsymbol{z}^{*}+d_{1} \frac{\boldsymbol{\lambda}}{\|\boldsymbol{\lambda}\|}\right)\right\|
\end{gathered}
$$

and $\theta$ is the preset penalty factor. Fig. 1 shows the approach of PBI. $\boldsymbol{\lambda}=\left(\lambda_{1}, \lambda_{2}, \cdots, \lambda_{M}\right)^{\top}$ is the weight vector, $\lambda_{i}$ satisfies $\lambda_{i} \geq 0$ and $\sum_{i=1}^{M} \lambda_{i}=1 . \boldsymbol{z}^{*}=\left(z_{1}^{*}, z_{2}^{*}, \cdots, z_{M}^{*}\right)^{\top}$ denotes the reference point. $H^{\prime}$ is the projection of $\mathbf{f}(\mathbf{x})$ onto the corresponding weight vector $\boldsymbol{\lambda}$. $d_{2}$ is the vertical distance between $\mathbf{f}(\mathbf{x})$ and $H^{\prime} . d_{1}$ is the straight distance from $\boldsymbol{z}^{*}$ to $H^{\prime}$.

\section{B. Normalization}

In the process of seeking the optimal solutions of MOPs, the scale of different objectives may be very different. The normalization operation can improve the performance of the solutions in the face of the above situation. In DTR-MOEA/D, all the objectives will be normalized and then projected. The normalization method adopted herein is expressed as follows:

$$
f_{i}^{\prime}(x)=\frac{f_{i}(x)-z_{i}^{*}}{f_{i}^{\max }(x)-z_{i}^{*}}
$$

where $f_{i}^{\prime}(x)$ is the normalized objective value of $f_{i}(x), \forall i \in$ $\{1,2, \cdots, M\}$, and $z^{*}=\left(z_{1}^{*}, z_{2}^{*}, \cdots, z_{M}^{*}\right)^{\top}$ denotes the ideal reference point. $f_{i}^{\max }(x)$ represents the maximum value of $f_{i}(x)$ among all the individuals generated in each iteration.

\section{Related Works}

In the past few years, MOEA/D has been broadly explored to solve various practical problems due to its excellent performance [43]. The theoretical research field has also witnessed the development boom of MOEA/D [44], and a large number of MOEA/D variants have appeared in the literature to deal with MOPs with various characteristics. The penalty factor incremental strategy was proposed to balance the diversity and convergence [45]. After this pioneering work, the dynamic adjustment strategy of penalty factor was paid great attention particularly in [2]. A unique weight vectors adjustment strategy was devised in [46]. Besides, an idea of hierarchical decomposition was adopted to assign subproblems to different hierarchies recently [47]. For the convenience of readers, we summarize the common improvement modules of the MOEA/D framework as follows.

Different reproductive operations affect the performance of population diversity and convergence. In the original MOEA/D, the simulated binary crossover (SBX) operator was selected to generate new individuals. It is gratifying that the differential evolution (DE) operators have been developed to embed into MOEA/D to improve the performance of the population in the evolutionary process [48], [49]. Previous experience was utilized to guide the selection of different DE operators [50]. An adaptive DE operator selection strategy based on the current population information was used to balance the population diversity and convergence [51]. Overall, the strategy of replacing the SBX operator with DE operator is greatly meaningful for the newly generated individuals in the aspect of overall population performance.

The selection range of mating and replacement has a significant impact on the population diversity and convergence. An adaptive neighborhood size selection mechanism was developed to balance the convergence and distribution of individuals [52]. Different neighborhood sizes were combined together to select the appropriate size according to the evolutionary performance of the population [53]. The proper neighborhood size was assigned to each sub-problem at different evolutionary stages in [54]. A novel adaptive replacement strategy was designed to enhance convergence and diversity [55]. It is worth affirming that the research on the size of mating and replacement greatly improves the performance of the algorithms while saving computing resources.

\section{DTR-MOEA/D}

Recent years have witnessed a growing number of MOEA/D variants that are applied for solving a wide variety of MOPs. The proposed DTR-MOEA/D, particularly developed for handing irregular MOPs, will be described in detail. Below, we first introduce the reference point dynamic transfer strategy. Second, we describe the diversity enhancement strategy. Then, the overall framework of DTR-MOEA/D is designed. At last, the computational complexity of DTR-MOEA/D is analyzed in detail.

\section{A. Reference Point Dynamic Transfer Strategy}

When solving an MOP, the POF shape of the MOP may be linear, convex, concave, or other shapes, which in most cases 


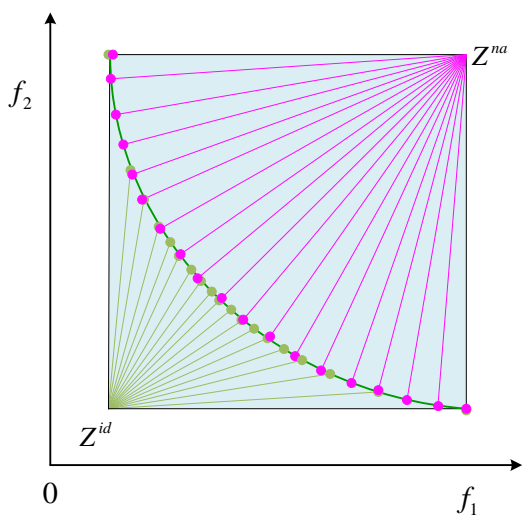

Fig. 2. Distribution of approximate solutions on convex POF with reference points $Z^{i d}$ and $Z^{\text {na }}$ respectively.
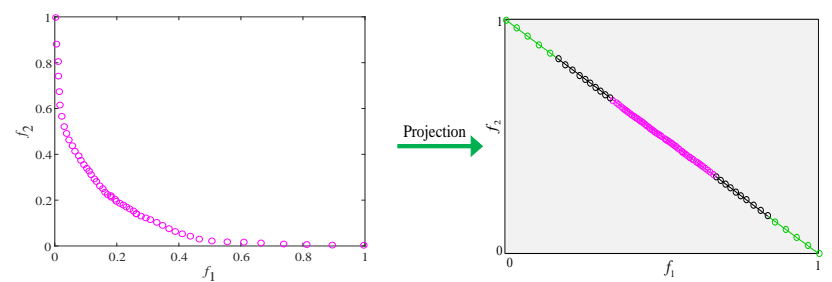

Fig. 3. Distribution of projected individuals in the case of two-objective.

is unknown in advance. Besides, on the convex POF with a sharp peak and long tail, the approximate solutions obtained by taking the ideal point as the reference point are densely distributed in the intermediate region and sparsely distributed at the two ends. However, the distribution of approximate solutions obtained by using the nadir point as the reference point is more uniform. Fig. 2 shows a two-objective case. According to the distribution of approximate solutions to judge the shape of the POF, and it is of great significance to select the appropriate reference point for solving this kind of problem. Under the guidance of this concept, we develop a dynamic transfer strategy of reference point based on the individual density relationship in different regions of the projection line segment or three-dimensional plane.

First, the individuals generated after each iteration are projected onto a two-dimensional line segment or a threedimensional plane. It is well known that the shape of the POF of an MOP can be convex, concave, mixed, and so on. It is difficult to accurately judge the density of the individuals which are approximately distributed on the irregular POF. In order to accurately judge the distribution of individuals from a mathematical point of view, we pioneer the concept of projection and zoning. The approximate individuals obtained by optimizing the MOP with two-objective are projected onto a line segment. We take the maximum of two objectives as the two endpoints of the line segment and divide the segment into six equal parts. The two segments at the center are marked as the central region, the two segments at the edges are marked as the edge region, and the remaining two segments are marked as the middle region, as shown in Fig. 3. The individuals projected onto the different regions are marked

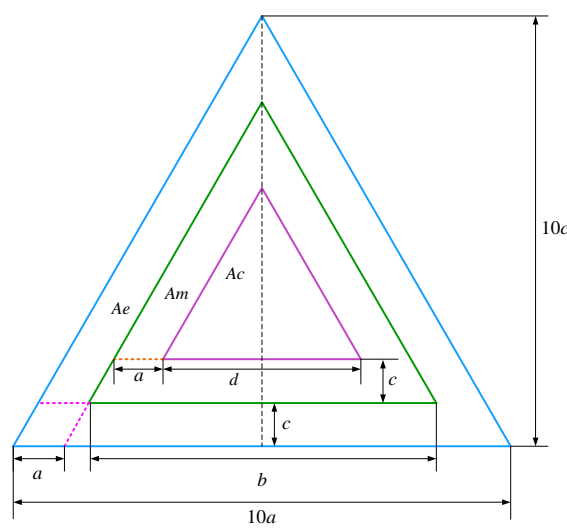

Fig. 4. Schematic diagram of area division.
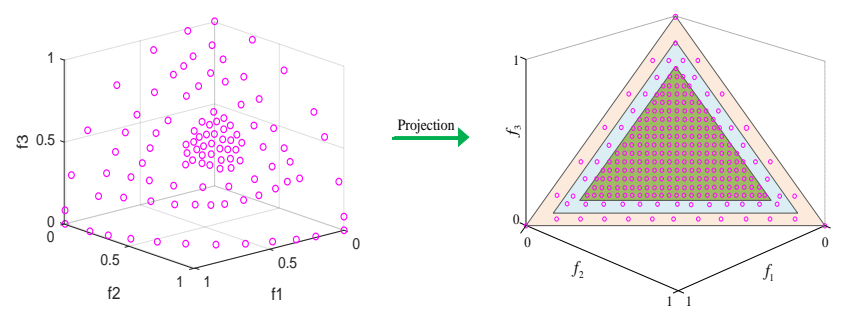

Fig. 5. Distribution of projected individuals in the case of three-objective.

with three different colors. An MOP with three-objective is taken as an example to illustrate the process of calculating individual density in different regions, as indicated in Fig. 4. Note that the side length of the edge triangle is preset. For the convenience of calculation, we set it to $10 a$. Other values are also available. For convergence, we present the relevant alphabetic symbols and the associated implications in Table I. The approximate individuals obtained by optimizing the MOP with three-objective are projected onto a three-dimensional plane. Note that for the convenience of calculation, the areas of different regions are not equal, which is different from the situation of the bi-objective case. The plane is divided into a central region, a middle region, and an edge region, as shown in Fig. 5. According to the number of individuals in different regions, the individual density of the region is calculated.

The individual density in different areas is calculated as follows:

$$
\begin{gathered}
A_{e}=\frac{1}{2}(10 a)^{2} \sin \left(\frac{1}{3} \pi\right)=25 \sqrt{3} a^{2} \\
\frac{9 c}{10 c}=\frac{\frac{b}{2}+a}{5 a}
\end{gathered}
$$

then we get

$$
b=7 a
$$

$$
A_{m}=\frac{1}{2} b^{2} \sin \left(\frac{1}{3} \pi\right)=\frac{1}{2}(7 a)^{2} \sin \left(\frac{1}{3} \pi\right)=\frac{49 \sqrt{3}}{4} a^{2} .
$$

Considering

$$
\frac{8 c}{10 c}=\frac{\frac{d}{2}+2 a}{5 a}
$$


TABLE I

ALPHABETIC SyMbOLS AND IMPLICATIONS

\begin{tabular}{c|c}
\hline Symbols & Meanings \\
\hline $10 a$ & Edge triangle side length \\
\hline $10 c$ & Edge triangle height \\
\hline$b$ & Middle triangle side length \\
\hline$d$ & Central triangle side length \\
\hline$A_{e}$ & Edge triangle area \\
\hline$A_{m}$ & Middle triangle area \\
\hline$A_{c}$ & Central triangle area \\
\hline$A_{e-m}$ & Edge region area \\
\hline$A_{m-c}$ & Middle region area \\
\hline$N_{e}$ & Number of individuals in edge region \\
\hline$N_{m}$ & Number of individuals in middle region \\
\hline$N_{c}$ & Number of individuals in central region \\
\hline$\rho_{e}$ & Edge region individual density \\
\hline$\rho_{m}$ & Middle region individual density \\
\hline$\rho_{c}$ & Central region individual density \\
\hline &
\end{tabular}

and therefore

$$
d=4 a
$$

$$
A_{c}=\frac{1}{2} d^{2} \sin \left(\frac{1}{3} \pi\right)=\frac{1}{2}(4 a)^{2} \sin \left(\frac{1}{3} \pi\right)=4 \sqrt{3} a^{2} .
$$

The edge region area is computed as

$$
A_{e-m}=A_{e}-A_{m}=25 \sqrt{3} a^{2}-\frac{49 \sqrt{3}}{4} a^{2}=\frac{51 \sqrt{3}}{4} a^{2} .
$$

The middle region area is derived from

$$
A_{m-c}=A_{m}-A_{c}=\frac{49 \sqrt{3}}{4} a^{2}-4 \sqrt{3} a^{2}=\frac{33 \sqrt{3}}{4} a^{2} .
$$

The individual density of the edge region can be calculated as

$$
\rho_{e}=\frac{N_{e}}{A_{e-m}}=\frac{4 \sqrt{3} N_{e}}{153 a^{2}} .
$$

The individual density of the middle region is given by

$$
\rho_{m}=\frac{N_{m}}{A_{m-c}}=\frac{4 \sqrt{3} N_{m}}{99 a^{2}} .
$$

The individual density of the central region can be obtained as

$$
\rho_{c}=\frac{N_{c}}{A_{c}}=\frac{\sqrt{3} N_{c}}{12 a^{2}} .
$$

Then, we have

$$
\begin{aligned}
& \rho_{\frac{m}{e}}=\frac{\rho_{m}}{\rho_{e}}=\frac{4 \sqrt{3} N_{m}}{99 a^{2}}\left(\frac{4 \sqrt{3} N_{e}}{153 a^{2}}\right)^{-1}=\frac{17}{11} \frac{N_{m}}{N_{e}} \\
& \rho_{\frac{c}{m}}=\frac{\rho_{c}}{\rho_{m}}=\frac{\sqrt{3} N_{c}}{12 a^{2}}\left(\frac{4 \sqrt{3} N_{m}}{99 a^{2}}\right)^{-1}=\frac{33}{16} \frac{N_{c}}{N_{m}} .
\end{aligned}
$$

Then, the dynamic transfer strategy of the reference point is formulated according to the individual density relationship of the two adjacent regions. If $\rho \frac{m}{e}>1$ and $\rho_{\frac{c}{m}}>1$, it indicates that the individuals in the population are sparsely distributed in the edge region, and the individual distribution density increases gradually from the edge region to the central region. The reference point should be changed from the ideal (nadir) point to the nadir (ideal) point. Otherwise, the reference point attribute remains unchanged.

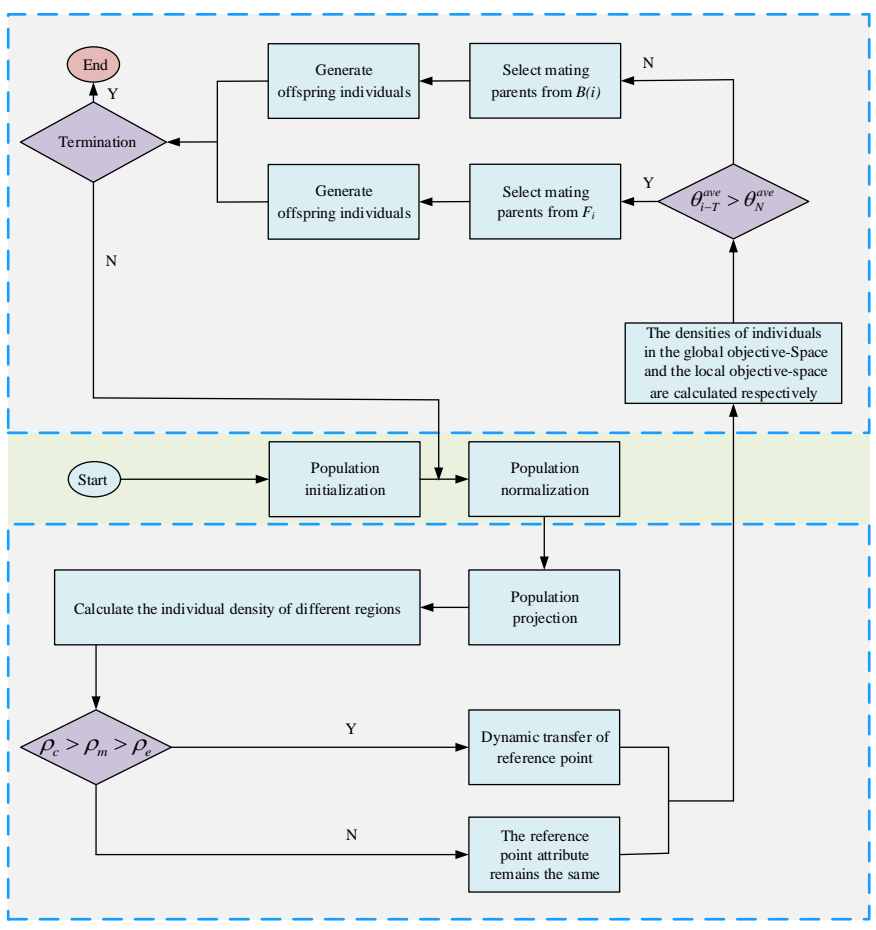

Fig. 6. The basic architecture of DTR-MOEA/D.

\section{B. Mating Selection Strategy Based on Individual Density in the Local Objective Space}

To further enhance evolutionary performance of the population, in this paper we put forward a novel diversity enhancement strategy guided by the relationship between the individual density knowledge in the global objective-space and the local objective-space. This paper is the first work that integrates the global objective-space individual density and local objective-space individual density for guiding mating parents selection. More specifically, we first calculate the minimum angle between each individual and the rest of the population in the global objective-space, labeled $\theta_{N}^{1}, \theta_{N}^{2}, \ldots, \theta_{N}^{N}$, respectively, and take the average of these $N$ angles as follows:

$$
\theta_{N}^{\text {ave }}=\frac{\theta_{N}^{1}+\theta_{N}^{2}+\cdots+\theta_{N}^{N}}{N}
$$

where $N$ denotes the number of the subproblems considered in DTR-MOEA/D.

Next, we calculate the minimum angle between each individual and the rest of the individuals in the local objectivespace, labeled $\theta_{i-T}^{1}, \theta_{i-T}^{2}, \ldots, \theta_{i-T}^{N}$, respectively, and take the average of these $T$ angles as follows:

$$
\begin{gathered}
\theta_{i-T}^{\text {ave }}=\frac{\theta_{i-T}^{1}+\theta_{i-T}^{2}+\cdots+\theta_{i-T}^{T}}{T} \\
\theta^{\overline{\text { ave }}}=\theta_{i-T}^{\text {ave }}-\theta_{N}^{\text {ave }}
\end{gathered}
$$

where $T$ denotes the number of the weight vectors in the neighborhood of each weight vector, and $i$ represents the $i$-th individual in the population.

At last, the selection range of the mating parents is determined according to the average value of the individual density 
in the global objective-space and the average value of the individual density in the local objective-space. If $\theta^{\overline{\mathrm{ave}}}<0$, it means that the distribution of individuals in the local objectivespace is denser and the similarity between individuals is higher than that in the global objective-space. It is reasonable to select individuals outside the neighborhood as mating parents that are conducive to increasing the diversity of the offspring population. Hence, the selection range of the mating parents can be constrained as follows:

$$
P= \begin{cases}B(i) & \text { if } \theta^{\overline{\text { ave }}} \geq 0 \\ F_{q} & \text { otherwise }\end{cases}
$$

$B(i)=\left\{i_{1}, i_{2}, \ldots, i_{T}\right\}$, where $\lambda^{i_{1}}, \lambda^{i_{2}}, \ldots, \lambda^{i_{T}}$ are the $T$ closest weight vectors to $\lambda^{i} . F_{q}$ represents a solution set that does not include individuals in $B(i)$. It is worth noting that we use the $\mathrm{DE} / \mathrm{rand} / 1$ operator to replace the SBX operator in the process of generating new individuals. When $\theta^{\overline{\text { ave }}}<0$, we need to select mating parents from $F_{q}$ to enhance the search ability in the process of population evolution.

\section{Framework of the Proposed DTR-MOEA/D}

Based on the MOEA/D-DE and inspired by the recently proposed MOEA/D-TPN, we propose the DTR-MOEA/D, as given in Fig. 6. The designed DTR-MOEA/D is mainly composed of the reference point dynamic transfer strategy and the diversity enhancement strategy. Specifically, for the reference point dynamic transfer strategy, we first calculate the individual density in different regions and then decide whether to carry out the reference point transfer operation according to $\rho_{\frac{m}{e}}$ and $\rho_{\frac{c}{m}}$. For the diversity enhancement strategy, we first calculate the individual density $\theta_{N}^{\text {ave }}$ in the global objectivespace and the individual density $\theta_{i-T}^{\text {ave }}$ in the local objectivespace respectively and then decide to select the appropriate mating parents from $B(i)$ or $F_{q}$ by judging the relationship between $\theta_{i-T}^{\text {ave }}$ and $\theta_{N}^{\text {ave }}$.

The pseudo-code of DTR-MOEA/D is given in Algorithm 1 , which can be divided into the following important steps. First, we initialize the population, reference point, and weight vectors. Besides, we straightforwardly elaborate the division of the projection region and the calculation of the individual density in different regions. Second, we compare the individual density in different regions. At last, new individuals are generated. The population and reference point are updated respectively.

\section{Computational Cost of One Generation of DTR-MOEA/D}

This subsection analyzes the computational complexity of our proposed DTR-MOEA/D. For clarity, it is necessary to set the following parameters: the number of objectives contained in the MOP is set to $M$. The population size is set to $N$. The number of the weight vectors in the neighborhood of each weight vector is set to $T$. The time complexity of updating the weight vectors is $O\left(N^{2}\right)$. The computational cost of updating an individual's neighborhood is $O(M N T)$, so the computational cost of updating the neighborhood of $N$ individuals is $O\left(M T N^{2}\right)$. It is worth noting that $T$ is usually a relatively small number. Therefore, the computational cost of DTR-MOEA/D is actually $O\left(M N^{2}\right)$.

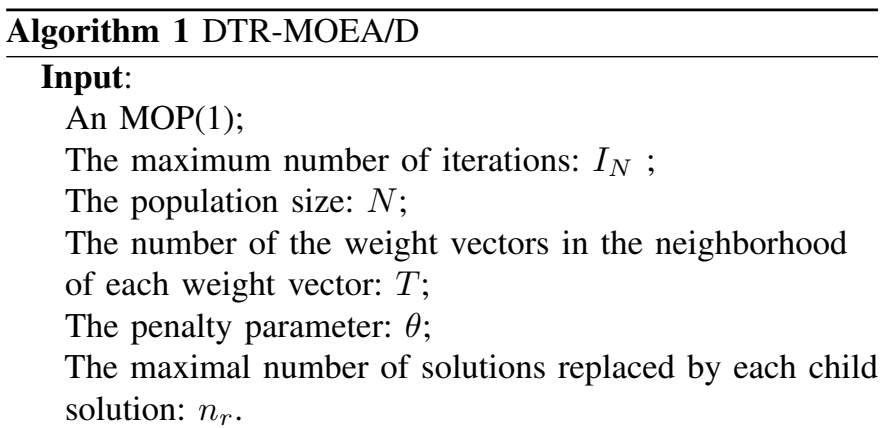

\section{Output:}

A set of solutions.

Step 1 Initialization:

Step 1.1 Generate an initial population $\mathbf{x}^{1}, \mathbf{x}^{2}, \ldots, \mathbf{x}^{N}$ by uniformly sampling from $\Omega$.

Step 1.2 Generate an initial reference point $\mathbf{z}^{*}$.

Step 1.3 Generate $N$ uniformly distributed weight vectors. Step 1.4 Determine the $T$ weight vectors closest to $\lambda^{i}$ by Euclidean distances $(i=1,2, \ldots, N)$.

\section{Step 2 Projection and Partitioning:}

/*For the two-objective optimization problem*/

Step 2.1 Calculate the number of individuals projected onto the interval of the different line segment.

/*For the three-objective optimization problem*/

Step 2.2 Calculate the area of the center, middle, and edge regions using equations (7)-(15).

Step 2.3 Calculate the density of individuals in the center, middle, and edge regions using equations (16)-(18).

Step 2.4 Calculate the density of individuals in the global objective-space using equation (21).

Step 2.5 Calculate the density of individuals in the local objective-space using equation (22).

\section{Step 3 Density Evaluation:}

Step 3.1 Determine the individual density relationship in the adjacent two regions of the central region, the middle region and the edge region using equations (19) and (20). Step 3.2 Determine the individual density relationship in the global objective-space and local objective-space using equation (23).

\section{Step 4 Update:}

For $i=1,2, \ldots, N$, do

Step 4.1 Select the appropriate mating parents according to equation (24).

Step 4.2 Use DE/rand/1 operator and polynomial mutation to generate new individuals.

Step 4.3 Use equations (19) and (20) to update the reference point.

Step 4.4 Update of solutions.

\section{Step 5 Stoping Criterion:}

If the stopping criterion is satisfied, then stop and output a set of non-dominated solutions. Otherwise, go to Step 2.

\section{Performance Verification of DTR-MOEA/D}

This section lists the experimental settings in detail and the comprehensive analysis of the experimental results. Five representative MOEAs with different characteristics are adopted 
to compare with the proposed DTR-MOEA/D for dealing with conventional MOPs and MOPs with irregular Pareto optimal front shapes.

\section{A. Test Problems and Compared Algorithms}

Three test suites consisting of MOPs with various attributes, e.g., DTLZ [56], WFG [57], and IMOP [58], are introduced to investigate the performance of DTR-MOEA/D on solving complex problems. DTLZ [56] and WFG [57] contain 16 widely used benchmark problems with linear, convex, concave, and discontinuous features. IMOP [58] is composed of 8 recently designed MOPs with irregular POF shapes. We compare the proposed DTR-MOEA/D with five representative or stateof-the-art MOEA/D variants, which include BCE-MOEA/D [1], ENS-MOEA/D [53], MOEA/D-AWA [36], MOEA/D-TPN [42], and MOEA/D-FRRMAB [59]. For clarity, we briefly describe the characteristics of each of the five compared algorithms as follows:

1) BCE-MOEA/D [1]: Pareto criterion and non-Pareto criterion promote the evolution of each other through a large number of information exchanges in the process of evolutionary optimization, and give full play to their respective advantages while compensates for each other's weaknesses.

2) ENS-MOEA/D [53]: $K$-fixed neighborhood sizes are selected as a pool of candidate individuals in ENSMOEA/D. Each subproblem is assigned a specific neighborhood size according to the candidate individuals' previous performances of generating improved solutions.

3) MOEA/D-AWA [36]: A novel initialization method of weight vector is proposed. The sparsity of each individual is calculated. The subproblems of the overcrowded regions are deleted and new subproblems are added to the sparse regions. In addition, the strategy of weight vector adjustment is carried out periodically.

4) MOEA/D-TPN [42]: The evolution process of the population is divided into two stages, and at the end of the first stage, whether to use the reversed scalar subproblem is decided by judging the density of the individuals. In addition, a niche guided update selection strategy is proposed to improve the population diversity.

5) MOEA/D-FRRMAB [59]: The adaptive DE operator selection strategy based on the bandit is integrated into the framework of MOEA/D. In the optimization procedure, according to the received credit values, the appropriate DE operator is selected to generate new individuals.

\section{B. Experimental Settings}

To ensure the fairness of comparison, the baseline algorithm in all the compared algorithms is replaced by MOEA/DDE [48], and these compared algorithms are denoted as BCE-MOEA/D-DE, AWA-MOEA/D-DE, TPN-MOEA/D-DE, MAB-MOEA/D-DE, and ENS-MOEA/D-DE, respectively. Other parameters are kept as the default values in their original references. The five compared MOEAs and the proposed DTRMOEA/D have the same population sizes and function evaluation times on each MOP. The population sizes and evolutionary
TABLE II

Settings of Test Problems

\begin{tabular}{c|c|c|c|c|c|c|c|c|c}
\hline Problem & $M$ & $D$ & $N$ & $I_{N}$ & Problem & $M$ & $D$ & $N$ & $I_{N}$ \\
\hline DTLZ1 & 3 & 7 & 200 & 300 & WFG6 & 2 & 22 & 150 & 200 \\
\hline DTLZ2 & 3 & 12 & 200 & 300 & WFG7 & 2 & 22 & 150 & 200 \\
\hline DTLZ3 & 3 & 12 & 200 & 300 & WFG8 & 2 & 22 & 150 & 200 \\
\hline DTLZ4 & 3 & 12 & 200 & 300 & WFG9 & 2 & 22 & 150 & 200 \\
\hline DTLZ5 & 3 & 12 & 200 & 300 & IMOP1 & 2 & 10 & 150 & 200 \\
\hline DTLZ6 & 3 & 12 & 200 & 300 & IMOP2 & 2 & 10 & 150 & 200 \\
\hline DTLZ7 & 3 & 22 & 200 & 300 & IMOP3 & 2 & 10 & 150 & 200 \\
\hline WFG1 & 2 & 22 & 150 & 200 & IMOP4 & 3 & 10 & 200 & 300 \\
\hline WFG2 & 2 & 22 & 150 & 200 & IMOP5 & 3 & 10 & 200 & 300 \\
\hline WFG3 & 2 & 22 & 150 & 200 & IMOP6 & 3 & 10 & 200 & 300 \\
\hline WFG4 & 2 & 22 & 150 & 200 & IMOP7 & 3 & 10 & 200 & 300 \\
\hline WFG5 & 2 & 22 & 150 & 200 & IMOP8 & 3 & 10 & 200 & 300 \\
\hline
\end{tabular}

generations of all the test MOPs in the experiment, as well as the number of objectives and decision variables contained in each MOP, are listed in Table II. $M, D, N$, and $I_{N}$ represent the number of objectives, the number of decision variables, the population size, and the iteration times of an MOP, respectively. The parameters of the comparative algorithms are set in the same manner as in the original literatures. In DTRMOEA/D, the penalty factor and the neighborhood size are set to $\theta=5$ and $T=20$, respectively. The $\mathrm{DE} / \mathrm{rand} / 1$ operator [60] and polynomial mutation operation [61] are applied to produce new individuals. The control parameters of $C R$ and $F$ are set to 1.0 and 0.5 , respectively. The distribution index and the mutation rate are set to $\eta=20$ and $p_{m}=\frac{1}{D}$, where $D$ is the number of decision variables, respectively.

\section{Performance Indicators}

To comprehensively evaluate the performance of the proposed DTR-MOEA/D and other five typical or state-of-the-art MOEAs on test problems from the aspects of convergence and diversity, two typical performance indicators, inverted generational distance (IGD) [62] and hypervolume (HV) [63], are employed in this paper.

1) IGD indicator: IGD needs to obtain a set of uniformly distributed points on the real POF of a test MOP as a reference set, which is denoted as $R_{S}$. In the process of population evolution, $A_{S}$ is a set of new approximate individuals generated after each iteration. The inverted generational distance from $R_{S}$ to $A_{S}$ is defined as

$$
\operatorname{IGD}\left(R_{S}, A_{S}\right)=\frac{\sum_{I_{x} \in R_{S}} d\left(I_{x}, A_{S}\right)}{\left|R_{S}\right|}
$$

where $d\left(I_{x}, A_{S}\right)$ is the specified minimum Euclidean distance between the individual $I_{x}$ and the individuals in $A_{S}$.

2) HV indicator: Hypervolume can evaluate the convergence and diversity of a set of individuals simultaneously, so it is often adopted as a performance indicator to evaluate the performance of MOEAs. The hypervolume is defined as

$$
\operatorname{HV}\left(N_{S}\right)=\operatorname{Leb}\left(U_{i=1}^{\left|N_{S}\right|} S_{i}^{V}\right)
$$

where $\operatorname{Leb}(\cdot)$ denotes the Lebesgue measure, $S_{i}^{V}$ is the supervolume of non-dominant solution and the reference point, 
TABLE III

Performance Comparison of the Competitive Algorithms Using IGD Mean and Standard Deviation Values on DTLZ, WFG, and IMOP TEST INSTANCES

\begin{tabular}{|c|c|c|c|c|c|c|}
\hline oblem & BCE-MOEA/D-DE & AWA-MOEA/D-DE & TPN-MOEA/D-DE & MAB-MOEA/D-DE & ENS-MOEA/D-DE & DTR-MOEA/D \\
\hline & $1.4551 \mathrm{E}-2(1.58 \mathrm{E}-4)+$ & $027 \mathrm{E}-1(1.22 \mathrm{E}-1)-$ & $3.5661 \mathrm{E}-2(5.08 \mathrm{E}-2)-$ & $3978 \mathrm{E}-2(2.85 \mathrm{E}-2)-$ & $2.4673 \mathrm{E}-1(5.13 \mathrm{E}-1)-$ & $2.6132 \mathrm{E}-2(3.18 \mathrm{E}-4)$ \\
\hline LZ2 & 86E-4)- & E-2)- & $2 \mathrm{E}-4) \approx$ & $E-4)-$ & 08E-4)- & $61 \mathrm{E}-4)$ \\
\hline TLZ3 & $.43 E+0)+$ & $6 \mathrm{E}+1)$ & $4603 \mathrm{E}+0(5.39 \mathrm{E}+0)-$ & & & $2.17 \mathrm{E}-3)$ \\
\hline DTLZ4 & $4.1286 \mathrm{E}-2(6.21 \mathrm{E}-4)+$ & 7.8842E-2(8.09E-2)- & 7.6631E-2(7.20E-2)- & $5.8351 \mathrm{E}-2(4.29 \mathrm{E}-3)+$ & $9.7495 \mathrm{E}-2(9.64 \mathrm{E}-2)-$ & $6.5164 \mathrm{E}-2(5.27 \mathrm{E}-2)$ \\
\hline TLZ5 & & .31E-3)- & & & & \\
\hline & $6 \mathbf{E}-5)+$ & E-4)+ & & & & \\
\hline TLZ7 & $707 \mathrm{E}-2(1.04 \mathrm{E}-1)-$ & .48E-1)- & $\mathrm{E}+0(9.34 \mathrm{E}-1)$ & E-2)- & $8.73 \mathrm{E}-2)$ & $.62 \mathrm{E}-3)$ \\
\hline FG1 & $1.0430 \mathrm{E}+0(5.60 \mathrm{E}-2)-$ & $.17 \mathrm{E}-1)-$ & $1.6291 \mathrm{E}+0(4.62 \mathrm{E}-1)-$ & $1.2021 \mathrm{E}+0(2.68 \mathrm{E}-2)-$ & $1.1728 \mathrm{E}+0(2.42 \mathrm{E}-2)-$ & $1.8725 \mathrm{E}-1(4.41 \mathrm{E}-3)$ \\
\hline WFG2 & $1.6810 \mathrm{E}-2(2.08 \mathrm{E}-3)+$ & $2.7531 \mathrm{E}-1(4.51 \mathrm{E}-2)-$ & $4.7855 \mathrm{E}-2(8.34 \mathrm{E}-3)-$ & $5.2351 \mathrm{E}-2(1.43 \mathrm{E}-2) \approx$ & $1.15 \mathrm{E}-2)-$ & $4.1573 \mathrm{E}-2(5.26 \mathrm{E}-4)$ \\
\hline FG3 & E-3)- & E-2)- & $-3)-$ & 3-2)- & -3)- & $52 \mathrm{E}-3)$ \\
\hline FG4 & E-3)- & E-2)- & 2E-2)- & E-2)- & $0 \mathrm{E}-3)$ & $4 \mathrm{E}-3(1.92 \mathrm{E}-4)$ \\
\hline WFG5 & $6.3770 \mathrm{E}-2(5.43 \mathrm{E}-4) \approx$ & $3 \mathrm{E}-1(5.42 \mathrm{E}-2)-$ & $6.8081 \mathrm{E}-2(1.74 \mathrm{E}-3)-$ & $9 \mathrm{E}-3)-$ & $32 \mathrm{E}-3)-$ & $51 \mathrm{E}-2(2.85 \mathrm{E}-3)$ \\
\hline & & $3.6335 \mathrm{E}-1(3.82 \mathrm{E}-2)-$ & $1.0559 \mathrm{E}-1(2.27 \mathrm{E}-2)-$ & $75 \mathrm{E}-2)-$ & $355 \mathrm{E}-2)-$ & $2(3.68 \mathrm{E}-2)$ \\
\hline & E-3)+ & E-2)- & $4 \mathrm{E}-3)+$ & (E-3)+ & $9 \mathrm{E}-3)+$ & $-2(6.55 \mathrm{E}-3)$ \\
\hline G8 & $E-3) \approx$ & E-2)- & E-3)- & E-2)- & $3 \mathrm{E}-2)-$ & $.28 E-4)$ \\
\hline WFG9 & IE-2)- & (E-2)- & 5E-2)- & (E-2)- & $6 \mathrm{E}-2)$ & $3.2813 \mathrm{E}-2(2.73 \mathrm{E}-2)$ \\
\hline IMOP1 & $300 \mathrm{E}-2(4.14 \mathrm{E}-3)-$ & $2 \mathrm{E}-2)-$ & $-3) \approx$ & 4 E-3)- & $6 \mathrm{E}-3)-$ & $26 \mathrm{E}-2(5.22 \mathrm{E}-3)$ \\
\hline MOP2 & (E-1)- & 9E-3)- & $5 \mathrm{E}-2)-$ & $\mathrm{E}-3) \approx$ & $.15 E-3)+$ & $3.1082 \mathrm{E}-2(2.53 \mathrm{E}-3)$ \\
\hline & $-3)-$ & E-1)- & & & $-3)-$ & $3(1.26 \mathrm{E}-3)$ \\
\hline & & & & & & $6.31 \mathrm{E}-4)$ \\
\hline & & $\mathrm{E}-2) \approx$ & & E-2)- & $8 \mathrm{E}-3)-$ & $33 \mathrm{E}-2(5.95 \mathrm{E}-4)$ \\
\hline IMOP6 & $2.2945 \mathrm{E}-2(6.10 \mathrm{E}-4)+$ & $.71 \mathrm{E}-3)-$ & $4308 \mathrm{E}-2(8.17 \mathrm{E}-4)+$ & $16 \mathrm{E}-4)-$ & $97 \mathrm{E}-4) \approx$ & $2.6172 \mathrm{E}-2(1.62 \mathrm{E}-4)$ \\
\hline IMOP7 & $2.8593 \mathrm{E}-2(2.86 \mathrm{E}-3)-$ & $7.6158 \mathrm{E}-1(2.48 \mathrm{E}-1)-$ & $8.1634 \mathrm{E}-2(3.92 \mathrm{E}-2)-$ & $5.2785 \mathrm{E}-2(4.43 \mathrm{E}-3)-$ & $1.3552 \mathrm{E}-1(2.55 \mathrm{E}-1)-$ & $2.3374 \mathrm{E}-2(8.26 \mathrm{E}-2)$ \\
\hline & $5.9826 \mathrm{E}-2(1.27 \mathrm{E}-3)-$ & & & & $1.7994 \mathrm{E}-1(1.05 \mathrm{E}-2)-$ & $5.3792 \mathrm{E}-2(7.16 \mathrm{E}-4)$ \\
\hline & & & $3 / 18 / 3$ & & & \\
\hline
\end{tabular}

and $N_{S}$ denotes a set of the non-dominant solutions. In the experiments, the reference point is set to $(1.1,1.1)^{\top}$ and $(1.1,1.1,1.1)^{\top}$ for bi-objective and tri-objective test cases, respectively.

Note that the larger the HV value (the lower the IGD value) of an MOEA on a test MOP, the better the performance of the MOEA. In order to make the experimental comparison more reliable, the results of each MOEA on each test MOP are obtained by executing 30 independent experimental runs, and the mean and standard deviation values are recorded. The statistically significant differences in IGD and HV values between each compared MOEA and the proposed DTRMOEA/D are evaluated using the Wilcoxon rank sum test [64] with a significance $\alpha$-level $(\alpha=0.05)$. The mathematical symbols "+ ", " $-"$, and " $\approx$ " are employed to denote that the indicator values obtained by each compared MOEA is significantly better, worse, and similar to that of proposed DTR-MOEA/D, respectively.

\section{Performance Comparisons}

1) Comparison on the DTLZ Test Problems: We first calculate the IGD performance indicator value of the designed DTR-MOEA/D and five other compared evolutionary algorithms on the DTLZ test suite. As reported in Table III, the proposed DTR-MOEA/D and BCE-MOEA/D-DE have attained inspiringly performance on DTLZ1-DTLZ7 MOPs. The DTR-MOEA/D on DTLZ2, DTLZ5, and DTLZ7 achieved the least mean inverted generational distance compared with BCE-MOEA/D-DE, AWA-MOEA/D-DE, TPNMOEA/D-DE, MAB-MOEA/D-DE, and ENS-MOEA/D-DE, respectively. We can find that the ENS-MOEA/D-DE gets the worst results on the seven MOPs compared with other algorithms. As previously discussed, the neighborhood size plays an important role in population diversity and convergence. Although there are several neighborhood sizes that can be selected by ENS-MOEA/D-DE, the neighborhood size can not be adjusted adaptively according to the evolution state of the population, which affects the performance of the population in the evolution process to some extent.

Furthermore, the $\mathrm{HV}$ indicator is used to evaluate the performance of the designed DTR-MOEA/D and the other five compared MOEAs on the DTLZ test suite. As shown in Table IV, our devised DTR-MOEA/D has achieved remarkably better performance than those MOEAs. Specifically, DTR-MOEA/D, BCE-MOEA/D-DE, AWA-MOEA/D-DE, TPN-MOEA/D-DE, MAB-MOEA/D-DE, and ENS-MOEA/D-DE obtain a better $\mathrm{HV}$ value in four, two, zero, one, zero, and zero out of the 7 test MOPs, respectively.

2) Comparison on the WFG Test Problems: The WFG test suite is widely adopted to test the performance of the MOEAs designated for tackling MOPs involving linear, convex, concave, mixed, degenerate, and disconnected Pareto optimal geometries. Table III presents the IGD value ( mean and standard deviation) of individuals obtained by DTR-MOEA/D and other popular and classical MOEA/D variants on solving MOPs from WFG1 to WFG9. Overall, our proposed DTR-MOEA/D has achieved the best performance among the five evolutionary algorithms under comparison in terms of IGD indicator. The DTR-MOEA/D wins on 7 test problems in terms of IGD out of 9 test MOPs in total. In contrast, BCE-MOEA/D-DE, AWAMOEA/D-DE, TPN-MOEA/D-DE, MAB-MOEA/D-DE, and ENS-MOEA/D-DE win one, zero, zero, zero, and one test problems in terms of IGD out of 9 test MOPs, respectively. We find that BCE-MOEA/D-DE achieves the least mean inverted generational distance on WFG2, the reason is that the dualcriterion evolution mechanism composed of Pareto criteri- 
TABLE IV

Performance Comparison of the Competitive Algorithms Using HV Mean and Standard Deviation Values on DTLZ, WFG, and IMOP TEST INSTANCES

\begin{tabular}{|c|c|c|c|c|c|c|}
\hline roblem & BCE-MO & AWA-MOEA/D-DE & TPN-MOEA/D-DE & MAB-MOEA/D-DE & ENS-MOEA/D-DE & EA/D \\
\hline & $7.54 \mathrm{E}-4)+$ & E-1(1.73E-1)- & $9 \mathrm{E}-1(1.17 \mathrm{E}-1)-$ & $.0245 \mathrm{E}-1(7.52 \mathrm{E}-2)-$ & $6.0975 \mathrm{E}-1(3.46 \mathrm{E}-1)-$ & $8.2517 \mathrm{E}-1(2.51 \mathrm{E}-2)$ \\
\hline LZ2 & $\mathrm{E}-4) \approx$ & E-2)- & $.43 E-4)+$ & $5 E-3) \approx$ & .27E-3)- & $(1.53 \mathrm{E}-4)$ \\
\hline OTLZ3 & .41E-1)- & $.9276 \mathrm{E}-1(1.69 \mathrm{E}-1)-$ & $2.6702 \mathrm{E}-1(2.41 \mathrm{E}-1)-$ & $-1)$ & $1252 \mathrm{E}-2(1.66 \mathrm{E}-1)-$ & $5.8351 \mathrm{E}-1(2.92 \mathrm{E}-3)$ \\
\hline DTLZ4 & $5.6594 \mathrm{E}-1(7.64 \mathrm{E}-4)-$ & $5.5691 \mathrm{E}-1(2.98 \mathrm{E}-2)-$ & $5.6010 \mathrm{E}-1(2.73 \mathrm{E}-2)-$ & $5.5590 \mathrm{E}-1(9.77 \mathrm{E}-4) \approx$ & $5.3805 \mathrm{E}-1(4.49 \mathrm{E}-2)-$ & $5.8164 \mathrm{E}-1(3.84 \mathrm{E}-3)$ \\
\hline TLZ5 & $7 \mathrm{E}-5) \approx$ & $6.30 \mathrm{E}-4)-$ & $.63 \mathrm{E}-5) \approx$ & 5)- & 93E-5)- & \\
\hline & & E-4)- & E-5)- & & & \\
\hline DTLZ7 & $.27 \mathrm{E}-2)+$ & $2.2812 \mathrm{E}-1$ & $.00 \mathrm{E}+0)-$ & $4 E-3)+$ & $2.4431 \mathrm{E}-1(4.76 \mathrm{E}-3)+$ & .61E-3) \\
\hline VFG1 & $2.3768 \mathrm{E}-1(1.97 \mathrm{E}-2)-$ & $2.7483 \mathrm{E}-1(3.88 \mathrm{E}-2)-$ & $2(9.53 \mathrm{E}-2)-$ & $5.75 \mathrm{E}-3)-$ & $1.8789 \mathrm{E}-1(5.26 \mathrm{E}-3)-$ & $4.9275 \mathrm{E}-1(7.22 \mathrm{E}-4)$ \\
\hline NFG2 & 37E-3)- & $5.5965 \mathrm{E}-1(1.28 \mathrm{E}-2)-$ & $6.0471 \mathrm{E}-1(6.35 \mathrm{E}-3)-$ & $9.99 \mathrm{E}-3)-$ & $6.1101 \mathrm{E}-1(8.53 \mathrm{E}-3)-$ & $6.2857 \mathrm{E}-1(4.16 \mathrm{E}-3)$ \\
\hline NFG3 & E-3)- & $E-2) \approx$ & $5 \mathrm{E}-3) \approx$ & DE-3)- & $-3) \approx$ & 53E-4) \\
\hline WFG4 & E-3)- & E-3)- & E-3)- & E-3)- & E-3)- & $.55 E-4)$ \\
\hline WFG5 & $.40 \mathrm{E}-4)-$ & 19E-2)- & 57E-3)- & $4 \mathrm{E}-3)-$ & & $.11 E-3)$ \\
\hline WFG6 & $2.9978 \mathrm{E}-1(1.95 \mathrm{E}-2)-$ & $2.0665 \mathrm{E}-1(1.80 \mathrm{E}-2)-$ & $2.9096 \mathrm{E}-1(1.26 \mathrm{E}-2)-$ & $.54 \mathrm{E}-2)-$ & 2)- & 64E-3) \\
\hline JFG7 & $-4)+$ & E-3)- & E-4)+ + & E-3)- & $9 \mathrm{E}-3)+$ & $26 \mathrm{E}-2)$ \\
\hline NFG8 & E-3)- & E-3)- & $E-3) \approx$ & 9E-2)- & $2 \mathrm{E}-2)-$ & $2 \mathrm{E}-2)$ \\
\hline WFG9 & $\mathrm{E}-2) \approx$ & $2.7788 \mathrm{E}-1$ & 7E-2)- & $3 \mathrm{E}-2)-$ & $3.2348 \mathrm{E}-1(1.90 \mathrm{E}-2)+$ & $3.2068 \mathrm{E}-1$ \\
\hline MOP1 & 2E-5)- & E-3)- & $4 \mathrm{E}-5)-$ & $8 \mathrm{E}-5)-$ & $32 \mathrm{E}-5)-$ & 5.31E-5) \\
\hline MOP2 & E-2)- & E-6)- & $-2)-$ & $-3)-$ & E-4)- & 5.33E-4) \\
\hline & E-4)- & E-2)- & $-4)-$ & & & 28E-3) \\
\hline & & & & & & 61E-4) \\
\hline DP5 & $3 E-2)+$ & E-3)- & E-3)- & & & $59 \mathrm{E}-3)$ \\
\hline 1OP6 & $34 \mathrm{E}-4)-$ & 84E-3)- & $.14 \mathrm{E}-4) \approx$ & $7.18 \mathrm{E}-4)-$ & $6.41 \mathrm{E}-4)-$ & $5.4126 \mathrm{E}-1(6.38 \mathrm{E}-4)$ \\
\hline IMOP7 & $5.3392 \mathrm{E}-1(1.05 \mathrm{E}-3)-$ & $1.4501 \mathrm{E}-1(1.12 \mathrm{E}-1)-$ & $4.6082 \mathrm{E}-1(6.85 \mathrm{E}-2)-$ & $5.1455 \mathrm{E}-1(2.29 \mathrm{E}-3)-$ & $4.7374 \mathrm{E}-1(1.28 \mathrm{E}-1)-$ & $5.3974 \mathrm{E}-1(3.52 \mathrm{E}-2)$ \\
\hline IMOP8 & $5.3215 \mathrm{E}-1(2.36 \mathrm{E}-3)-$ & & & & $4.6632 \mathrm{E}-1(4.86 \mathrm{E}-3)-$ & $5.3581 \mathrm{E}-1(6.27 \mathrm{E}-2)$ \\
\hline & & & & $1 / 20 / 3$ & & \\
\hline
\end{tabular}

on and non-Pareto criterion has advantages in dealing with problems involving convex and disconnected Pareto optimal geometries.

The HV indicator values obtained by DTR-MOEA/D and five other compared algorithms on different test problems are listed in Table IV. As seen, the devised DTR-MOEA/D works quite efficiently. Additionally, we also present the statistical significance of DTR-MOEA/D and other five algorithms. We can observe that, despite that DTR-MOEA/D does not achieve the maximum $\mathrm{HV}$ value for all test problems such as WFG7 and WFG9, the statistical results of most test problems are significantly better than those of other algorithms.

3) Comparison on the IMOP Test Problems: Besides the above two benchmark test suites used for numerical comparison, more attention has been paid to solve other multiobjective testing problems with irregular Pareto front shapes. IMOP test suite is a typical MOP suite, which contains of eight MOPs with highly irregular Pareto optimal front shapes. The Pareto optimal fronts of IMOP1 and IMOP2 are strong convex and strong concave with long tails and sharp peaks, respectively. IMOP3 has a discontinuous Pareto front. IMOP4-IMOP8 have highly irregular Pareto fronts, respectively. Table III shows the mean and standard deviation IGD values of the solutions obtained by six algorithms in 30 independent runs. Clearly, the designed DTR-MOEA/D is statistically significantly better than the other five comparative algorithms. It wins on 5 out of 8 test problems in terms of the IGD values. Among the six evolutionary algorithms, however, AWA-MOEA/D-DE, TPNMOEA/D-DE, and MAB-MOEA/D-DE do not perform well when solving IMOP1-IMOP8 test problems. BCE-MOEA/DDE only achieved the minimum inverted generational distance on IMOP4 and IMOP6. We can easily find that TPNMOEA/D-DE performs well on IMOP4 and IMOP6. From these IGD results, we can ascertain that the devised DTRMOEA/D has superior to the other algorithms. We further demonstrate the performance of different algorithms under the HV indicator. As shown in Table IV, our DTR-MOEA/D has achieved better performance than the other algorithms on all the IMOP test problems.

As can be observed from Tables III and IV, the devised DTR-MOEA/D generally performs better than the other compared evolutionary algorithms. More specifically, the DTRMOEA/D achieve the minimum inverted generational distance value in 15 out of the 24 test problems, followed by BCE-MOEA/D-DE and ENS-MOEA/D-DE achieving 7 and 2 best results, respectively. In terms of the Wilcoxon rank sum test, the proportion of test problems where our proposed DTR-MOEA/D has inspiringly better IGD values than BCE-MOEA/D-DE, AWA-MOEA/D-DE, TPN-MOEA/D-DE, MAB-MOEA/D-DE, and ENS-MOEA/D-DE is 14/24, 21/24, 18/24, 19/24, and 20/24, respectively. For HV, our DTRMOEA/D achieve the maximum HV value in 18 out of the 24 test MOPs, followed by BCE-MOEA/D-DE, TPN-MOEA/DDE, and ENS-MOEA/D-DE achieving 4, 1, and 1 best results, respectively. Additionally, the proportion of test MOPs where the DTR-MOEA/D has inspiringly better $\mathrm{HV}$ values than BCE-MOEA/D-DE, AWA-MOEA/D-DE, TPN-MOEA/D-DE, MAB-MOEA/D-DE, and ENS-MOEA/D-DE is $17 / 24,22 / 24$, $17 / 24,20 / 24$, and $18 / 24$, respectively.

In order to intuitively observe the performance of the designed DTR-MOEA/D and the other comparative algorithms, Fig. 7 clearly depicts the solutions with the lowest IGD obtained by different evolutionary algorithms on DTLZ2, WFG1, WFG4, IMOP1, IMOP3, and IMOP5. As illustrated in Fig. 7, it is not difficult to observe that the solutions obtained by our devised DTR-MOEA/D on various multiobjective test 

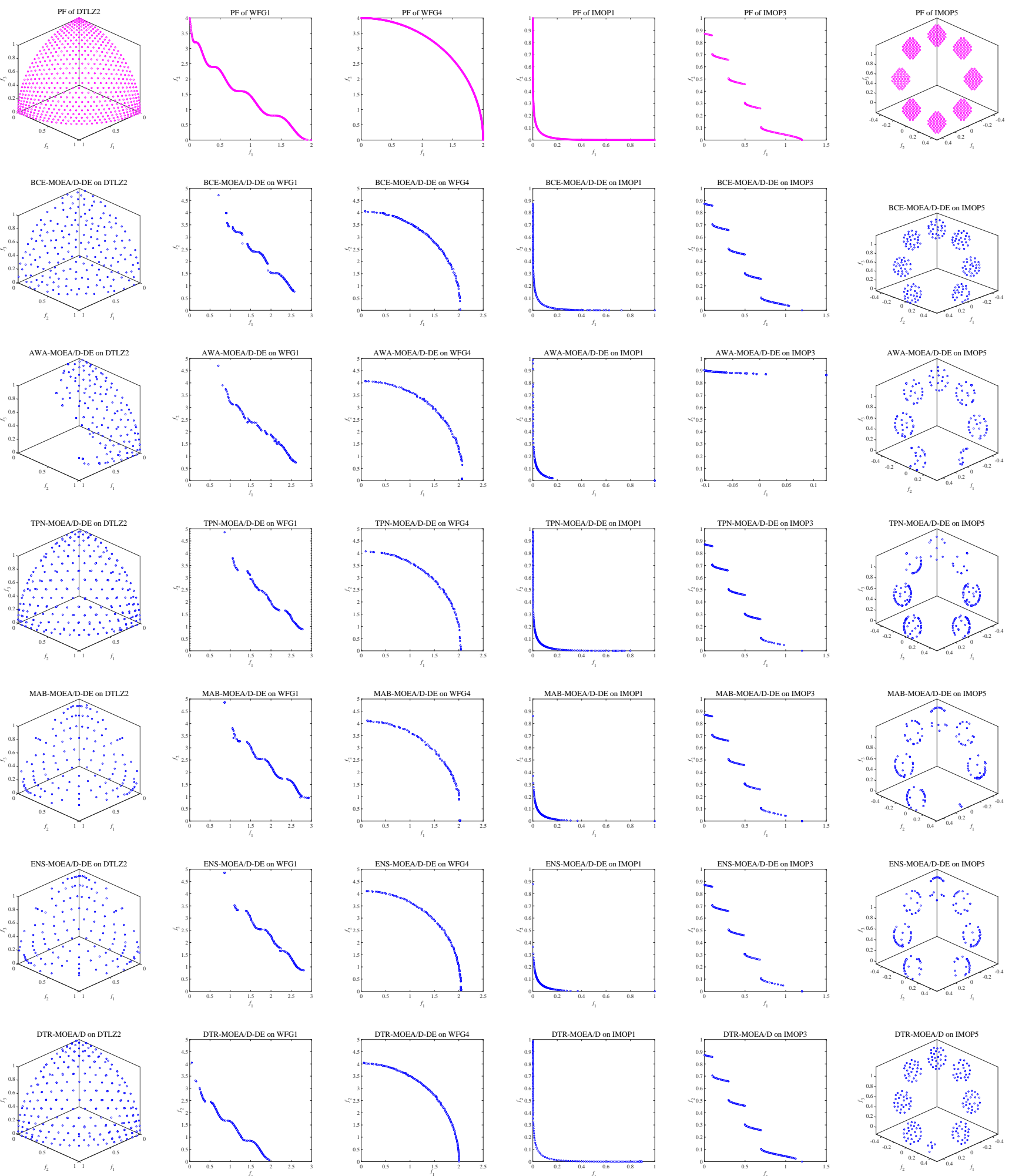

Fig. 7. Pareto fronts and the final solutions with the lowest IGD value among 30 independent runs obtained by BCE-MOEA/D-DE, AWA-MOEA/D-DE, TPN-MOEA/D-DE, MAB-MOEA/D-DE, ENS-MOEA/D-DE, and DTR-MOEA/D on DTLZ2, WFG1, WFG4, IMOP1, IMOP3, and IMOP5. 
problems can be well distributed over the entire Pareto optimal fronts. For the WFG1 test problem, which has a mixed convex/concave POF, the solutions of BCE-MOEA/D-DE, AWAMOEA/D-DE, TPN-MOEA/D-DE, MAB-MOEA/D-DE, and ENS-MOEA/D-DE on WFG1 after the same function evaluation times can not converge to the POF, and the distribution of solutions is not uniform, showing the phenomenon of dense in the middle and sparse at both ends. The reason why DTRMOEA/D can achieve superior performance on WFG1 is lie in the dynamic transfer strategy of reference point and the diversity enhancement strategy based on local objectivespace knowledge. These two measures also ensure that the solutions obtained by DTR-MOEA/D on other test problems can be distributed in the whole POF while guaranteeing the convergence.

\section{E. Discussions}

Although the proposed DTR-MOEA/D has attained the best overall performance among all the comparative algorithms used in this paper, it does not always perform satisfactorily. As shown in Fig. 7, although DTR-MOEA/D achieves the best performance among the six algorithms on WFG1, the obtained non-dominated individuals are not well distributed on the whole POF. This is also the case for IMOP1. The poor performance in extreme regions shows that although the reference point transfer strategy and the diversity enhancement strategy based on the knowledge in the local objective-space can promote the uniform distribution of individuals to a certain extent, they still can not solve the strong convex or strong concave problem well. How to ensure the uniform distribution of individuals in extreme regions is one of the future research directions. In addition, it is worthwhile to emphasize that the dynamic transfer strategy of reference point guided by the density of individuals in different regions can better ensure the diversity of individuals in solving MOPs with irregular Pareto front shape compared with the two-stage strategy. As is clearly seen from the statistical results in Tables III and IV, TPN-MOEA/D-DE performed better than DTR-MOEA/D in 3 and 2 of the 24 test problems, respectively. The TPNMOEA/D-DE with the ideal point as the reference point would decide whether to change the reference point when the evolution generation reaches $0.7 I_{N}$. This strategy is not conducive to maintaining the diversity of the population when solving MOPs with concave, mixed, and other irregular Pareto optimal front shapes.

We further compare the IGD and HV values of DTRMOEA/D and MAB-MOEA/D-DE on three test suites. The statistical results show that DTR-MOEA/D is significantly better than MAB-MOEA/D-DE in solving MOPs. In the process of population evolution, MAB-MOEA/D-DE randomly selected mating parents from a specific population, and there was no reference to the individual density information in the evolution process. Additionally, MAB-MOEA/D-DE only uses DE operator adaptive strategy and hence can not guarantee the uniform distribution of individuals in the whole irregular POF. One can see that, in general, our proposed DTR-MOEA/D also outperforms BCE-MOEA/D-DE, AWA-MOEA/D-DE, and ENS-MOEA/D-DE on 24 multiobjective test problems.

\section{CONCLusions}

In comparison to existing evolutionary algorithms, there are some spotlights in our work. First, the reference point dynamic transfer strategy is developed based on the density relationship of individuals in different mapping regions. Second, the diversity enhancement strategy is based on the relationship between the individual density in the local objective-space and that in the global objective-space. We further systematically integrate the above two strategies into the MOEA/D-DE framework to propose the final DTR-MOEA/D. Via experiments, the proposed DTR-MOEA/D has achieved noticeably better performance than the state-of-the-art MOEAs.

The proposed DTR-MOEA/D has demonstrated that the reference point dynamic transfer strategy and the diversity enhancement strategy are promising strategies for solving MOPs. However, further investigation of applying the above two strategies to other MOEAs is still desirable, especially on MOPs with time-varying decision variables or time-varying number of objectives. Additionally, it will be very meaningful for us to use DTR-MOEA/D to solve problems in the industrial field. For example, the urban wastewater treatment process is a multi-objective dynamic optimization process which contains multiple conflicting objectives. DTR-MOEA/D can be used to solve the conflict between the three objectives of aeration energy consumption, pumping energy consumption, and effluent quality in the wastewater treatment process, so as to achieve the purpose of reducing energy consumption and improving effluent quality. This is of great practical significance for saving energy and realizing the recycling of wastewater.

\section{REFERENCES}

[1] M. Li, S. Yang, and X. Liu, "Pareto or non-Pareto: Bi-criterion evolution in multiobjective optimization," IEEE Trans. Evol. Comput., vol. 20, no. 5, pp. 645-665, Oct. 2016.

[2] J. Qiao, H. Zhou, C. Yang, and S. Yang, "A decomposition-based multiobjective evolutionary algorithm with angle-based adaptive penalty," Appl. Soft. Comput., vol. 74, pp. 190-205, Jan. 2019.

[3] K. Zhang, G. G. Yen, and Z. He, "Evolutionary algorithm for knee-based multiple criteria decision making," IEEE Trans. Cybern., vol. 51, no. 2, pp. 722-735, Feb. 2021.

[4] Y. Tian, X. Zhang, R. Cheng, C. He, and Y. Jin, "Guiding evolutionary multiobjective optimization with generic front modeling," IEEE Trans. Cybern., vol. 50, no. 3, pp. 1106-1119, Mar. 2020.

[5] M. Li and X. Yao, "What weights work for you? Adapting weights for any Pareto front shape in decomposition-based evolutionary multiobjective optimisation," Evol. Comput., vol. 28, no. 2, pp. 227-253, Jun. 2020.

[6] R. Cheng, M. Li, K. Li, and X. Yao, "Evolutionary multiobjective optimization-based multimodal optimization: Fitness landscape approximation and peak detection," IEEE Trans. Evol. Comput., vol. 22, no. 5, pp. 692-706, Oct. 2018.

[7] C. He, L. Li, Y. Tian, X. Zhang, R. Cheng, Y. Jin, and X. Yao, “Accelerating large-scale multiobjective optimization via problem reformulation," IEEE Trans. Evol. Comput., vol. 23, no. 6, pp. 949-961, Dec. 2019.

[8] M. Li, S. Yang, and X. Liu, "Diversity comparison of Pareto front approximations in many-objective optimization," IEEE Trans. Cybern., vol. 44, no. 12, pp. 2568-2584, Dec. 2014.

[9] M. Li, S. Yang, and X. Liu, "Bi-goal evolution for many-objective optimization problems," Artif. Intell., vol. 228, pp. 45-65, Nov. 2015.

[10] Z. Fan, W. Li, X. Cai, H. Li, C. Wei, Q. Zhang, K. Deb, and E. Goodman, "Push and pull search for solving constrained multi-objective optimization problems," Swarm Evol. Comput., vol. 44, pp. 665-679, Feb. 2019

[11] Y. Xiang, Y. Zhou, M. Li, and Z. Chen, "A vector angle-based evolutionary algorithm for unconstrained many-objective optimization," IEEE Trans. Evol. Comput., vol. 21, no. 1, pp. 131-152, Feb. 2017. 
[12] H. Zhou and J. Qiao, "Multiobjective optimal control for wastewater treatment process using adaptive MOEA/D," Appl. Intell., vol. 49, no. 3, pp. 1098-1126, 2019.

[13] D. Wang, M. Ha, and J. Qiao, "Data-driven iterative adaptive critic control towards an urban wastewater treatment plant," IEEE Trans. Ind. Electron., vol. 68, no. 8, pp. 7362-7369, Aug.2021.

[14] Z. Fan, Y. You, X. Cai, H. Zheng, G. Zhu, W. Li, A. Gary, K. Deb, and E. Goodman, "Analysis and multi-objective optimization of a kind of teaching manipulator," Swarm Evol. Comput., vol. 50, pp. 100554, Nov. 2019.

[15] X. Zhang, Y. Zhou, Q. Zhang, V.V. Lee, and M. Li, "Problem specific MOEA/D for barrier coverage with wireless sensors," IEEE Trans. Cybern., vol. 47, no. 11, pp. 3854-3865, Nov. 2017.

[16] W.A. Albukhanajer, J.A. Briffa, and Y. Jin, "Evolutionary multiobjective image feature extraction in the presence of noise," IEEE Trans. Cybern., vol. 45, no. 9, pp. 1757-1768, Sept. 2015.

[17] H. Wang, Y. Jin, and J. Janse, "Data-driven surrogate-assisted multiobjective evolutionary optimization of a trauma system," IEEE Trans. Evol. Comput., vol. 20, no. 6, pp. 939-952, Dec. 2016.

[18] H. Zhang, D. Yue, W. Yue, K. Li, and M. Yin, "MOEA/D-based probabilistic PBI approach for risk-based optimal operation of hybrid energy system with intermittent power uncertainty," IEEE Trans. Syst., Man, Cybern., Syst.,, vol. 51, no. 4, pp. 2080-2090, Apr. 2021.

[19] A. Zhou, B. Qu, H. Li, S.Z. Zhao, P.N. Suganthan, and Q. Zhang, "LIBEA: A Lebesgue indicator-based evolutionary algorithm for multiobjective optimization," Swarm Evol. Comput., vol. 1, no. 1, pp. 32-49, Mar. 2011.

[20] S. Yang, M. Li, X. Liu, and J. Zheng, "A grid-based evolutionary algorithm for many-objective optimization," IEEE Trans. Evol. Comput., vol. 17 , no. 5, pp. 721-736, Oct. 2013.

[21] M. Li, S. Yang, and X. Liu, "Shift-based density estimation for Paretobased algorithms in many-objective optimization," IEEE Trans. Evol. Comput., vol. 18, no. 3, pp. 348-365, Jun. 2014.

[22] K. Deb, A. Pratap, S. Agarwal, and T.A.M.T. Meyarivan, "A fast and elitist multiobjective genetic algorithm: NSGA-II," IEEE Trans. Evol. Comput., vol. 6, no. 2, pp. 182-197, Apr. 2002.

[23] E. Zitzler, M. Laumanns, and L. Thiele, "SPEA2: Improving the strength Pareto evolutionary algorithm," TIK-report, vol. 103, 2001.

[24] H. Wang, L. Jiao, and X. Yao, "Two_Arch2: An improved two-archive algorithm for many-objective optimization," IEEE Trans. Evol. Comput., vol. 19, no. 4, pp. 524-541, Aug. 2015.

[25] W. Fang, L. Zhang, S. Yang, J. Sun, and X. Wu, "A multiobjective evolutionary algorithm based on coordinate transformation," IEEE Trans. Cybern., vol. 49, no. 7, pp. 2732-2743, July. 2019.

[26] X. Zhang, Y. Tian, R. Cheng, and Y. Jin, "An efficient approach to nondominated sorting for evolutionary multiobjective optimization," IEEE Trans. Evol. Comput., vol. 19, no. 2, pp. 201-213, Mar. 2014.

[27] E. Zitzler and S. Künzli, "Indicator-based selection in multiobjective search," in Proc. 8th Int. Conf. Parallel Problem Solving Nat., Birmingham, U.K., 2004, pp. 832-842.

[28] Y. Tian, R. Cheng, X. Zhang, F. Cheng, and Y. Jin, "An indicator-based multiobjective evolutionary algorithm with reference point adaptation for better versatility," IEEE Trans. Cybern., vol. 22, no. 4, pp. 609-622, Aug. 2018.

[29] S. Jiang, J. Zhang, Y.S. Ong, A.N. Zhang, and P.S. Tan, "A simple and fast hypervolume indicator-based multiobjective evolutionary algorithm," IEEE Trans. Cybern., vol. 45, no. 10, pp. 2202-2213, Oct. 2015.

[30] K. Shang, H. Ishibuchi, M. Zhang, and Y. Liu, "A new R2 indicator for better hypervolume approximation," in Proc. IEEE Congr. Evol. Comput. (CEC), Rio de Janeiro, Brazil, July. 2018, pp. 745-752.

[31] Y. Liu, J. Liu, T. Li, and Q. Li, "An R2 indicator and weight vector-based evolutionary algorithm for multi-objective optimization," Soft Comput., pp. 1-22, Aug. 2019.

[32] W. Hong, K. Tang, A. Zhou, H. Ishibuchi, and X. Yao, "A scalable indicator-based evolutionary algorithm for large-scale multiobjective optimization," IEEE Trans. Evol. Comput., vol. 23, no. 3, pp. 525-537, June. 2019.

[33] Q. Zhang and H. Li, "MOEA/D: A multiobjective evolutionary algorithm based on decomposition," IEEE Trans. Evol. Comput., vol. 11, no. 6, pp. 712-731, Dec. 2007.

[34] Z. Fan, W. Li, X. Cai, H. Huang, Y. Fang, Y. You, J. Mo, C. Wei, and E. Goodman, "An improved epsilon constraint-handling method in MOEA/D for CMOPs with large infeasible regions," Soft Comput., vol. 23, no. 23, pp. 12491-12510, Feb. 2019.

[35] X. Ma, Y. Yu, X. Li, Y. Qi, and Z. Zhu, "A survey of weight vector adjustment methods for decomposition-based multiobjective evolutionary algorithms," IEEE Trans. Evol. Comput., vol. 24, no. 4, pp. 634-649, Aug. 2020.

[36] Y. Qi, X. Ma, F. Liu, L. Jiao, J. Sun, and J. Wu, "MOEA/D with adaptive weight adjustment," Evol. Comput., vol. 22, no. 2, pp. 231-264, 2014.

[37] R. Cheng, Y. Jin, M. Olhofer, and B. Sendhoff, "A reference vector guided evolutionary algorithm for many-objective optimization," IEEE Trans. Evol. Comput., vol. 20, no. 5, pp. 773-791, Oct. 2016.

[38] X. Ma, F. Liu, Y. Qi, L. Li, L. Jiao, X. Deng, X. Wang, B. Dong, Z. Hou, Y. Zhang, and J. Wu, "MOEA/D with biased weight adjustment inspired by user preference and its application on multi-objective reservoir flood control problem," Soft Comput., vol. 20, no. 12, pp. 4999-5023, Dec. 2016.

[39] K. Li, K. Deb, Q. Zhang, and S. Kwong, "An evolutionary manyobjective optimization algorithm based on dominance and decomposition," IEEE Trans. Evol. Comput., vol. 19, no. 5, pp. 694-716, Oct. 2015.

[40] M. Asafuddoula, H.K. Singh, and T. Ray, "An enhanced decompositionbased evolutionary algorithm with adaptive reference vectors," IEEE Trans. Cybern., vol. 48, no. 8, pp. 2321-2334, Aug. 2018.

[41] Z. Wang, Q. Zhang, H. Li, H. Ishibuchi, and L. Jiao, "On the use of two reference points in decomposition based multiobjective evolutionary algorithms," Swarm Evol. Comput., vol. 34, pp. 89-102, Feb. 2019.

[42] S. Jiang and S. Yang, "An improved multiobjective optimization evolutionary algorithm based on decomposition for complex Pareto fronts," IEEE Trans. Cybern., vol. 46, no. 2, pp. 421-437, Feb. 2016.

[43] X. Zuo, X. Liu, Q. Zhang, W. Li, X. Wan, and X. Zhao, "MOEA/D with linear programming for double row layout problem with center-islands," IEEE Trans. Cybern., in press.

[44] Z. Fan, Y. Fang, W. Li, X. Cai, C. Wei, and E. Goodman, "MOEA/D with angle-based constrained dominance principle for constrained multiobjective optimization problems," Appl. Soft. Comput., vol. 74, pp. 621633, Jan. 2019

[45] S. Yang, S. Jiang, and Y. Jiang, "Improving the multiobjective evolutionary algorithm based on decomposition with new penalty schemes," Soft Comput., vol. 21, no. 16, pp. 4677-4691, Feb. 2016.

[46] C. Zhang, K.C. Tan, L.H. Lee, and L. Gao, "Adjust weight vectors in MOEA/D for bi-objective optimization problems with discontinuous Pareto fronts," Soft Comput., vol. 22, no. 12, pp. 3997-4012, Apr. 2018.

[47] H. Xu, W. Zeng, D. Zhang, and X. Zeng, "MOEA/HD: a multiobjective evolutionary algorithm based on hierarchical decomposition," IEEE Trans. Cybern., vol. 49, no. 2, pp. 517-526, Feb. 2019.

[48] H. Li and Q. Zhang, "Multiobjective optimization problems with complicated Pareto sets, MOEA/D and NSGA-II," IEEE Trans. Evol. Comput., vol. 13, no. 2, pp. 284-302, Apr. 2009.

[49] X. Cai, Z. Meng, Z. Fan, and Q. Zhang, "A constrained decomposition approach with grids for evolutionary multiobjective optimization," IEEE Trans. Evol. Comput., vol. 22, no. 4, pp. 564-577, Aug. 2018.

[50] S.M. Venske, R.A. Goncalves, and M.R. Delgado, "ADEMO/D: Multiobjective optimization by an adaptive differential evolution algorithm," Neurocomputing., vol. 127, pp. 65-77, Mar. 2014.

[51] Y. Xie, J. Qiao, D. Wang, and B. Yin, "A novel decomposition-based multiobjective evolutionary algorithm using improved multiple adaptive dynamic selection strategies," Inf. Sci., vol. 556, pp. 472-494, May. 2021.

[52] J. Zou, Y. Zhang, S. Yang, Y. Liu, and J. Zheng, "Adaptive neighborhood selection for many-objective optimization problems," Appl. Soft. Comput., vol. 64, pp. 186-198, Mar. 2018.

[53] S.Z. Zhao, P.N. Suganthan, and Q. Zhang, "Decomposition-based multiobjective evolutionary algorithm with an ensemble of neighborhood sizes," IEEE Trans. Evol. Comput., vol. 16, no. 3, pp. 442-446, June. 2012.

[54] S. Zhang, L. Zheng, L. Liu, S. Zheng, and Y. Pan, "Decompositionbased multi-objective evolutionary algorithm with mating neighborhood sizes and reproduction operators adaptation," Soft Comput., vol. 21, no. 21, pp. 6381-6392, June. 2017

[55] Z. Wang, Q. Zhang, A. Zhou, M. Gong, and L. Jiao, "Adaptive replacement strategies for MOEA/D," IEEE Trans. Cybern., vol. 46 , no. 2, pp. 474-486, Feb. 2016.

[56] K. Deb, L. Thiele, M. Laumanns, and E. Zitzler, "Scalable multiobjective optimization test problems," in Proc. IEEE Congr. Evol. Comput. (CEC), May. 2002, pp. 825-830.

[57] S. Huband, P. Hingston, L. Barone, and L. While, "A review of multiobjective test problems and a scalable test problem toolkit," IEEE Trans. Evol. Comput., vol. 10, no. 5, pp. 477-506, Oct. 2006.

[58] Y. Tian, C. He, R. Cheng, and X.Y. Zhang, "A multistage evolutionary algorithm for better diversity preservation in multiobjective optimization," IEEE Trans. Syst., Man, Cybern., Syst.,, in press. 
[59] K. Li, A. Fialho, S. Kwong, and Q. Zhang, "Adaptive operator selection with bandits for a multiobjective evolutionary algorithm based on decomposition," IEEE Trans. Evol. Comput., vol. 18, no. 1, pp. 114130, Feb. 2014

[60] K. Price, R.M. Storn, and J.A. Lampinen, "Differential evolution: a practical approach to global optimization." Secaucus, NJ, USA:Springer, 2005.

[61] K. Deb and M. Goyal, "A combined genetic adaptive search (GeneAS) for engineering design," Comput. Sci. Informat., vol. 26, no. 4, pp. 30 45, 1996.

[62] E. Zitzler, L. Thiele, M. Laumanns, C.M. Fonseca, and V.G. Da Fonseca, "Performance assessment of multiobjective optimizers: An analysis and review," IEEE Trans. Evol. Comput., vol. 7, no. 2, pp. 117-132, Apr. 2003.

[63] E. Zitzler and L. Thiele, "Multiobjective evolutionary algorithms: a comparative case study and the strength Pareto approach," IEEE Trans. Evol. Comput., vol. 3, no. 4, pp. 257-271, Nov. 1999.

[64] M.P. Fay and M.A. Proschan, "Wilcoxon-Mann-Whitney or t-test? On assumptions for hypothesis tests and multiple interpretations of decision rules," Statistics surveys, vol. 4, no. 1, pp. 1-39, Apr. 2010.

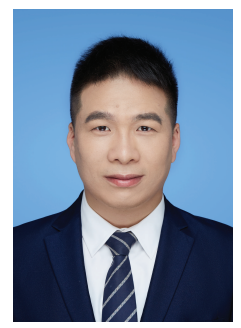

Yingbo Xie received the M.S. degree in control engineering from Henan Polytechnic University, Jiaozuo, China, in 2018. He is currently pursuing the Ph.D. degree in control science and engineering with the Beijing University of Technology, Beijing, China. His research interests include computational intelligence, evolutionary transfer optimization, and transfer learning.

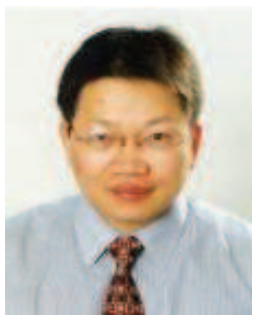

Shengxiang Yang (Senior Member, IEEE) received the PhD degree from Northeastern University, Shenyang, China in 1999 . He is currently a Professor in Computational Intelligence and Deputy Director of the Institute of Artificial Intelligence, School of Computer Science and Informatics, De Montfort University, Leicester, U.K. He has over 340 publications with an $\mathrm{H}$-index of 61 according to Google Scholar. His current research interests include evolutionary computation, swarm intelligence, artificial neural networks, data mining and data stream mining, and relevant real-world applications. He serves as an Associate Editor/Editorial Board Member of a number of international journals, such as the IEEE Transactions on Evolutionary Computation, IEEE Transactions on Cybernetics, Information Sciences, Enterprise Information Systems, and CAAI Transactions on Intelligence Technology.

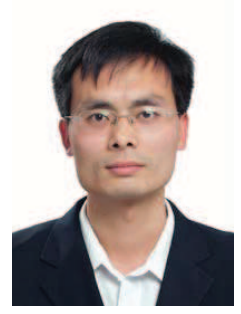

Ding Wang (Senior Member, IEEE) received the $\mathrm{Ph} . \mathrm{D}$. degree in control theory and control engineering from the Institute of Automation, Chinese Academy of Sciences, Beijing, China, in 2012. He is currently a Professor with the Faculty of Information Technology, Beijing University of Technology, Beijing. He has authored/coauthored over 120 journal and conference papers and four monographs. His current research interests include adaptive critic control with industrial applications, reinforcement learning, and intelligent systems. Prof. Wang currently serves as an Associate Editor for IEEE TRANSACTIONS ON SYSTEMS, MAN, AND CYBERNETICS: SYSTEMS, Neural Networks, International Journal of Robust and Nonlinear Control, Neurocomputing, and Acta Automatica Sinica.

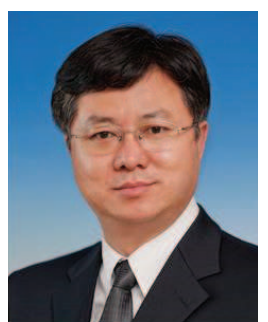

Junfei Qiao (Senior Member, IEEE) received the B.S. and M.S. degrees in control engineering from Liaoning Technical University, Fuxin, China, in 1992 and 1995, respectively, and the Ph.D. degree from Northeastern University, Shenyang, China, in 1998. He is currently a Professor with the Faculty of Information Technology, Beijing University of Technology, Beijing, China, where he is also the Director of the Beijing Key Laboratory of Computational Intelligence and Intelligent Systems and the Beijing Laboratory of Smart Environmental Protection. His current research interests include computational intelligence, intelligent systems, self-adaptive systems, and process control.

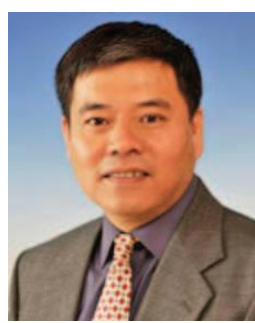

Baocai Yin (Member, IEEE) received the B.S., M.S., and Ph.D. degrees from the Dalian University of Technology, Dalian, China, in 1985, 1988, and 1993, respectively. $\mathrm{He}$ is currently a Professor with the Beijing Key Laboratory of Multimedia and Intelligent Software Technology, Beijing University of Technology, Beijing, China. His research interests include digital multimedia, multifunctional perception, virtual reality, and computer graphics. He is the vice chairman of ACM Beijing. 